\title{
A six-beam method to measure turbulence statistics using ground-based wind lidars
}

\author{
A. Sathe, J. Mann, N. Vasiljevic, and G. Lea \\ DTU Wind Energy, Ris $\emptyset$ campus, Roskilde, Denmark \\ Correspondence to: A. Sathe (amsat@dtu.dk)
}

Received: 22 September 2014 - Published in Atmos. Meas. Tech. Discuss.: 10 October 2014

Revised: 4 January 2015 - Accepted: 19 January 2015 - Published: 12 February 2015

\begin{abstract}
A so-called six-beam method is proposed to measure atmospheric turbulence using a ground-based wind lidar. This method requires measurement of the radial velocity variances at five equally spaced azimuth angles on the base of a scanning cone and one measurement at the centre of the scanning circle, i.e.using a vertical beam at the same height. The scanning configuration is optimized to minimize the sum of the random errors in the measurement of the second-order moments of the components $(u, v, w)$ of the wind field. We present this method as an alternative to the so-called velocity azimuth display (VAD) method that is routinely used in commercial wind lidars, and which usually results in significant averaging effects of measured turbulence. In the VAD method, the high frequency radial velocity measurements are used instead of their variances. The measurements are performed using a pulsed lidar (WindScanner), and the derived turbulence statistics (using both methods) such as the $u$ and $v$ variances are compared with those obtained from a reference cup anemometer and a wind vane at $89 \mathrm{~m}$ height under different atmospheric stabilities. The measurements show that in comparison to the reference cup anemometer, depending on the atmospheric stability and the wind field component, the six-beam method measures between 85 and $101 \%$ of the reference turbulence, whereas the VAD method measures between 66 and $87 \%$ of the reference turbulence.
\end{abstract}

\section{Introduction}

Wind lidars are being used significantly for wind energy applications. They measure mean wind speeds with great accuracy, and are very useful tools in the measurement of wind profiles (Smith et al., 2006; Kindler et al., 2007; Peña et al.,
2009; Wagner et al., 2011). New recommended practices are being defined for wind resource assessments (Clifton et al., 2013). However their use in measuring atmospheric turbulence has not yet been established, particularly with the commercial lidars (Sathe et al., 2011b). The main reason is that for a commercial lidar, the measured lidar data is processed using the so-called velocity azimuth display (VAD) method, where the measurements of the radial velocity (also called the line-of-sight velocity) at different azimuth angles are combined to deduce the wind field components. For the mean wind speed estimation, the VAD method produces negligible errors. For turbulence statistics the VAD method produces significant systematic errors (Sathe et al., 2011b; Sathe and Mann, 2012) mainly due to two reasons; one is the filtering of the smaller scales due to the large size of the probe volume within which the radial velocity is measured, and second is the contamination by the two-point correlation between the components of the wind field.

In this article we present a so-called six-beam method that significantly improves the measurement of turbulence in comparison to the VAD method. This method uses the variances of the radial velocities from six different lidar beams, five of which are at equally spaced azimuth angles on the base of a scanning cone and one beam is vertical. These variances are then combined in order to deduce the second-order moments of the wind field. A framework for this method was originally proposed by Lhermitte (1969), which was used by Wilson (1970) and Kropfli (1986) for radar studies, and subsequently by Eberhard et al. (1989) and Mann et al. (2010) for lidar studies of turbulence measurements. In their studies only the covariances were estimated, either by combining several measurements of the radial velocity variances from several lidar beams (Eberhard et al., 1989) at equally spaced 
azimuth angles and one elevation angle, or by using only two lidar beams (Mann et al., 2010). In the present work, six beams are used, five at an elevation angle of $45^{\circ}$ and one vertical that enable us to also deduce the variances.

The ideas to measure turbulence using remote sensing instruments have evolved, albeit slowly, since the pioneering works on radar meteorology (Lhermitte, 1962; Browning and Wexler, 1968). Based on the VAD scanning, Lhermitte (1969) was the first (to our knowledge) to suggest a technique of deducing turbulence using the measurements of the variance of the radial velocity. Subsequently Wilson (1970) was the first to conduct an experiment using a pulsed Doppler radar and deducing turbulence in the convective boundary layer $(0.1-1.3 \mathrm{~km})$. Only turbulence scales larger than the pulse volume but smaller than the scanning circle could be measured since all the data from a single scan was used. Also, no comparison with any reference instrument was carried out, and hence, the reliability of the radar measurements could not be verified. Kropfli (1986) extended the study of Wilson (1970) to also include the turbulence scales larger than the scanning circle by using the data from multiple scans. Although the method was developed for Doppler radar studies, it could also be used for Doppler lidar studies. Eberhard et al. (1989) was the first to perform turbulence studies using a lidar following the methods of Wilson (1970); Kropfli (1986). Gal-Chen et al. (1992) also used the variances of the radial velocities to deduce turbulence, but with a different scanning configuration. In all of the aforementioned studies with a Doppler lidar (or radar), the probe length was quite significant (of the order of $100 \mathrm{~m}$ ), which perhaps was the reason to restrict these studies to the convective boundary layer. However if the turbulence measurements were desired close to the ground then they would be subjected to a significant amount of probe volume averaging. It was perhaps this reason that the focus on turbulence research with lidars shifted to understanding the probe volume averaging effect and providing potential solutions to compensate for it (Frehlich, 1994, 1997; Frehlich et al., 1994, 1998, 2006, 2008; Frehlich and Cornman, 2002; Frehlich and Kelley, 2008; Banakh et al., 1995a, b, 1996, 1999, 2010; Banakh and Smalikho, 1997a, b; Banakh and Werner, 2005; Smalikho, 1995; Smalikho et al., 2005; Mann et al., 2010; Branlard et al., 2013). Even with the development of the modern lidar systems, where the probe lengths have shrunk to about $30 \mathrm{~m}$ for a pulsed lidar, significant amount of averaging still remains in the turbulence measurements within the surface layer, where the wind turbines operate (Mann et al., 2009, 2010; Sjöholm et al., 2009; Sathe et al., 2011b; Sathe and Mann, 2012). A detailed review of the state of the art with respect to turbulence measurements using ground-based wind lidars can be found in Sathe and Mann (2013).

Unfortunately within the wind energy sector, turbulence measurements are being deduced using the VAD scanning method, which results in a significant amount of filtering of turbulence, and contamination by the two-point correla-

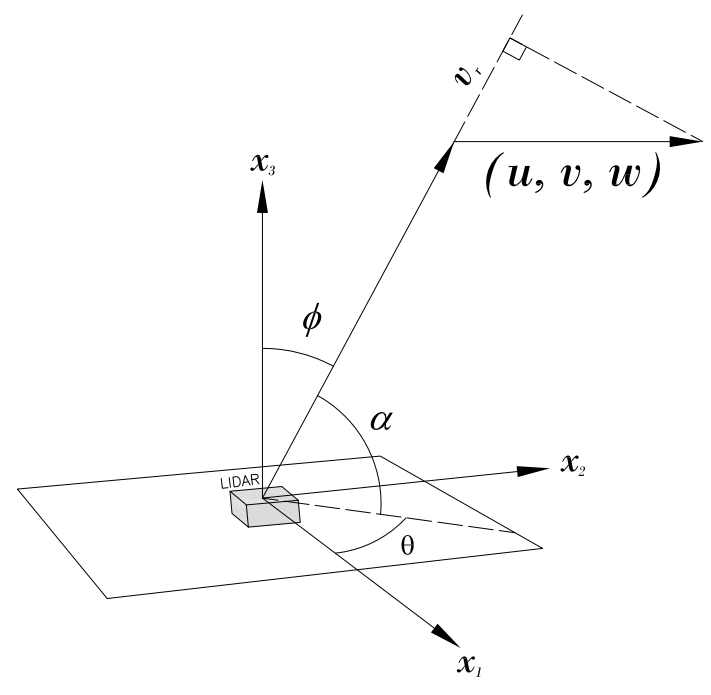

Figure 1. Coordinate system of a lidar.

tion between the components of the wind field (Sathe et al., 2011b). In this work we attempt to significantly improve the turbulence measurements compared to those obtained by the VAD method, by extending the previously developed ideas of using the radial velocity variances (Lhermitte, 1969; Wilson, 1970; Kropfli, 1986; Eberhard et al., 1989; Mann et al., 2010), but restricting them to using only six beams.

The structure of this article is divided into the following sections. Section 2 gives a detailed explanation of the six-beam technique. The optimum six-beam configuration, which is one of the main contributions of this article is also described in detail. In order to verify our method, turbulence measurements using the pulsed lidar WindScanner were performed and compared with a reference cup anemometer at a height of $89 \mathrm{~m}$. The site description for the measurements is given in Sect. 3, whereas the results are described in Sect. 4. Discussions and conclusions are made in Sects. 5 and 6, respectively.

\section{Theory of six-beam configuration}

The instantaneous velocity field is characterized as a vector $\boldsymbol{v}=(u, v, w)$, and turbulence is characterized as the components of the Reynolds stress tensor,

$\mathbf{R}=\left[\begin{array}{ccc}\left\langle u^{\prime 2}\right\rangle & \left\langle u^{\prime} v^{\prime}\right\rangle & \left\langle u^{\prime} w^{\prime}\right\rangle \\ \left\langle v^{\prime} u^{\prime}\right\rangle & \left\langle v^{\prime 2}\right\rangle & \left\langle v^{\prime} w^{\prime}\right\rangle \\ \left\langle w^{\prime} u^{\prime}\right\rangle & \left\langle w^{\prime} v^{\prime}\right\rangle & \left\langle w^{\prime 2}\right\rangle\end{array}\right]$,

where the diagonal terms are the variances of the respective wind field components and the off-diagonal terms are the covariances, \langle\rangle denote ensemble average, and ' denotes fluctuations around the average. 
As shown in Fig. 1, at a given instant of time if we assume that a lidar measures at a point, and that the lidar beam is inclined at a certain zenith angle $\phi$ (in some literature the complement of $\phi$ is used, which is called as the elevation angle $\alpha=90^{\circ}-\phi$ ) from the vertical axis, and makes an azimuth angle $\theta$ with respect to the axes in the horizontal plane, then the radial velocity (also called as the line-of-sight velocity) can be mathematically written as,

$v_{\mathrm{r}}\left(\phi, \theta, d_{\mathrm{f}}\right)=\boldsymbol{n}(\phi, \theta) \cdot \boldsymbol{v}\left(\boldsymbol{n}(\phi, \theta) d_{\mathrm{f}}\right)$,

where $v_{\mathrm{r}}$ is the radial velocity measured at a point, $\boldsymbol{n}=$ $(\cos \theta \sin \phi, \sin \theta \sin \phi, \cos \phi)$ is the unit directional vector for a given $\phi$ and $\theta$, and $d_{\mathrm{f}}$ is the distance at which the measurement is obtained. In Eq. (2), we have implicitly assumed that $v_{\mathrm{r}}$ is positive for the wind going away from the lidar axis, the coordinate system is right-handed, and $u$ is aligned with the $x_{1}$ axis in a horizontal plane, i.e. from west to east. In reality, a lidar never receives backscatter from exactly a point, but from all over the physical space. Fortunately the transverse dimensions of a lidar beam are much smaller than the longitudinal dimensions, and for all practical purposes we can consider that the backscatter is received only along the lidar beam axis. Mathematically the radial velocity can be represented as the convolved signal,

$\widetilde{v}_{\mathrm{r}}\left(\phi, \theta, d_{\mathrm{f}}\right)=\int_{-\infty}^{\infty} \varphi(s) \boldsymbol{n}(\phi, \theta) \cdot \boldsymbol{v}\left(\boldsymbol{n}(\phi, \theta)\left(d_{\mathrm{f}}+s\right)\right) \mathrm{d} s$,

where $\widetilde{v}_{\mathrm{r}}$ is the weighted average radial velocity, $\varphi(s)$ is any weighting function integrating to one that depends on the type of lidar, i.e. a continuous wave (c-w) lidar or a pulsed lidar, and $s$ is the distance along the beam from the measurement point of interest. From simple geometrical considerations the radial velocity variance can be written as a function of the components of $\mathbf{R}$ (Lhermitte, 1969; Eberhard et al., 1989),

$$
\begin{aligned}
\left\langle v_{\mathrm{r}}^{\prime 2}\right\rangle= & \left\langle u^{\prime 2}\right\rangle \sin ^{2} \phi \cos ^{2} \theta+\left\langle v^{\prime 2}\right\rangle \sin ^{2} \phi \sin ^{2} \theta+\left\langle w^{\prime 2}\right\rangle \cos ^{2} \phi \\
& +2\left\langle u^{\prime} v^{\prime}\right\rangle \sin ^{2} \phi \sin \theta \cos \theta+2\left\langle u^{\prime} w^{\prime}\right\rangle \sin \phi \cos \phi \cos \theta \\
& +2\left\langle v^{\prime} w^{\prime}\right\rangle \sin \phi \cos \phi \sin \theta,
\end{aligned}
$$

where $\left\langle v_{\mathrm{r}}^{\prime 2}\right\rangle$ is the radial velocity variance. From Eq. (4) we can see that for a given $\theta$ and $\phi$, if we have six measurements of $\left\langle v_{\mathrm{r}}^{\prime 2}\right\rangle$ then there are six unknowns to be determined, which in a matrix form can be written as,

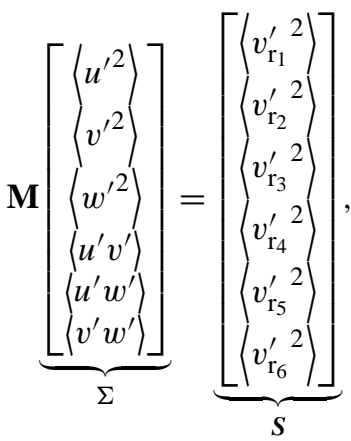

where $\Sigma$ is a vector of the components of $\mathbf{R}$ (because $\mathbf{R}$ is symmetric, we only need six components), $\mathbf{M}$ is a $6 \times 6 \mathrm{ma}-$ trix of the coefficients of $\Sigma$ that consist of different combinations of $\theta$ and $\phi$ (see Eq. 4), and $S$ is a vector of measurements of $\left\langle v_{\mathrm{r}}^{\prime 2}\right\rangle$ at different $\theta$ and $\phi$ (where the suffices denote measurements from beam 1 to 6 ). In principle we can then estimate $\Sigma$ using the relation $\Sigma=\mathbf{M}^{-1} \boldsymbol{S}$, where ${ }^{-1}$ denotes matrix inverse. It is interesting to know beforehand whether the measurements from the six beams on only one zenith angle are adequate, i.e. whether we can have six $\theta$ s and only one $\phi$.

From fundamental algebra we understand that Eq. (5) will have a finite solution if and only if $\operatorname{det} \mathbf{M} \neq 0$, where det denotes the determinant of a matrix. In other words $\mathbf{M}$ should not be a degenerate matrix. From the properties of determinants we know that if any two rows (or columns) of a matrix are identical then its determinant is zero. Also, if the elements of any row (or column) are increased (or decreased) by equal multiples of the corresponding elements of any other row (or column), the value of determinant is unchanged. If we use only one $\phi$ at different $\theta$, and add the first two columns of $\mathbf{M}$, we get the first and the third columns of $\mathbf{M}$ to be multiples of each other, which according to the property of determinants implies $\operatorname{det} \mathbf{M}=0$. Thus $\mathbf{M}$ becomes degenerate if we use only one $\phi$, and thus need $\left\langle v_{\mathrm{r}}^{\prime 2}\right\rangle$ measurements from more than one $\phi$.

We are then confronted with the challenge of obtaining an optimum combination of $\theta$ and $\phi$. Measured $S$ is stochastic, and the random error of $\Sigma$ will depend on the particular choice of the $\theta \mathrm{s}$ and $\phi \mathrm{s}$. We thus choose the objective function such that the sum of the random errors of the components of $\Sigma$ are minimized. For simplicity, we neglect the probe volume filtering effect in the derivation of the optimum combination, but including that will not change the optimum configuration.

It is to be noted that in Eq. (5), we have neglected the random error in the lidar estimate of the radial velocity. For the particular lidar used in this work the uncorrelated noise in the velocity estimation is exceedingly small and will not have any effect for the analysis presented in this paper. The very low noise level can be seen from plots of spectra in Figs. 2, 5, and 7 of Sathe and Mann (2012). 


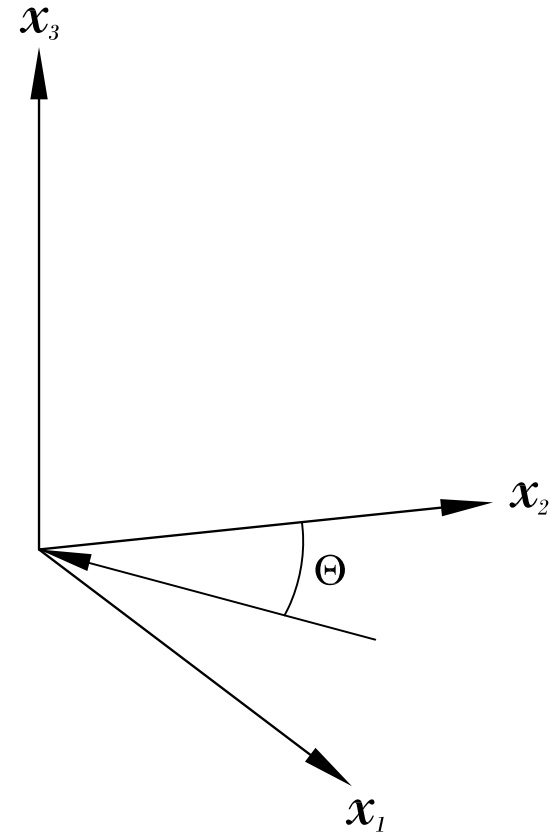

Figure 2. Standard meteorological convention of depicting the mean wind direction.

\subsection{Formulation of the objective function}

Equation (4) is valid for the mean wind direction aligned in the $x_{1}$ direction. Following standard meteorological conventions, let us consider the mean wind direction to be at an angle $\Theta$ with respect to the north, i.e. $x_{2}$ axis as shown in Fig. 2. At first we derive an objective function for the wind aligned with the $x_{1}$ axis, and then extend the derivation to the coordinate system aligned with the mean wind direction.

\subsubsection{Mean wind aligned with the $x_{1}$ axis}

If we consider that $\delta \Sigma$ is the random error on $\Sigma$, and $\delta S$ is the random error on $S$, then Eq. (5) can be written as,

$\mathbf{M}(\Sigma+\delta \Sigma)=S+\delta S$,

$\Sigma+\delta \Sigma=\mathbf{M}^{-1}(\boldsymbol{S}+\delta \boldsymbol{S})$

We can thus write,

$\delta \Sigma=\mathbf{M}^{-1} \delta S$.

If we consider the sum of the error variances $\left\langle\delta \Sigma^{\mathrm{T}} \boldsymbol{\delta} \Sigma\right\rangle$, where ${ }^{\mathrm{T}}$ denotes matrix transpose, then the objective is to minimize the sum of the error variances of the components of $\Sigma$. Taking the transpose and multiplying by Eq. (7) we get,

$$
\begin{aligned}
\delta \Sigma \cdot \delta \Sigma=\delta \Sigma^{\mathrm{T}} \boldsymbol{\delta} \Sigma & =\left(\mathbf{M}^{-1} \boldsymbol{\delta} \boldsymbol{S}\right)^{\mathrm{T}}\left(\mathbf{M}^{-1} \boldsymbol{\delta} \boldsymbol{S}\right) \\
& =\boldsymbol{\delta} \boldsymbol{S}^{\mathrm{T}}\left(\mathbf{M}^{-1 \mathrm{~T}} \mathbf{M}^{-1}\right) \boldsymbol{\delta} \boldsymbol{S}
\end{aligned}
$$

The task now is to simplify Eq. (8) such that it can be represented as a function of $\theta$ and $\phi$ only. If we assume that the random errors in the variances of the radial velocities are independent of each other, and that the error variance for each radial velocity variance is $\left\langle\epsilon_{\mathrm{s}}^{2}\right\rangle$, we get,

$\frac{\left\langle\boldsymbol{\delta} \Sigma^{\mathrm{T}} \boldsymbol{\delta} \Sigma\right\rangle}{\left\langle\epsilon_{\mathrm{s}}^{2}\right\rangle}=\operatorname{Tr}\left(\mathbf{M}^{-1} \mathbf{M}^{-1 \mathrm{~T}}\right)$,

where $\operatorname{Tr}$ is the trace of a matrix. The objective function is to minimize Eq. (9).

\subsubsection{Coordinate system aligned with the mean wind direction}

In order to align the coordinate system with the mean wind direction, we need to apply coordinate transformations on any tensors that are defined in the original coordinate system. The vector $v$ rotated in the mean wind direction has to be multiplied by a transformation matrix $\mathbf{T}$ given as,

$\mathbf{T}=\left[\begin{array}{ccc}-\sin \Theta & -\cos \Theta & 0 \\ \cos \Theta & -\sin \Theta & 0 \\ 0 & 0 & 1\end{array}\right]$.

In the coordinate system aligned with the mean wind direction, we then get in matrix form,

$\mathbf{R}_{\mathrm{r}}=\mathbf{T R T}^{\mathrm{T}}$,

where $\mathbf{R}_{\mathrm{r}}$ is the Reynolds stress tensor in a coordinate system aligned with the mean wind direction. If we denote $\Sigma_{\mathrm{r}}$ as the vector of the components of $\mathbf{R}_{\mathrm{r}}$, then we can write,

$\Sigma_{\mathrm{r}}=\underbrace{\left[\begin{array}{cccccc}\sin ^{2} \Theta & \cos ^{2} \Theta & 0 & \sin 2 \Theta & 0 & 0 \\ \cos ^{2} \Theta & \sin ^{2} \Theta & 0 & -\sin 2 \Theta & 0 & 0 \\ 0 & 0 & 1 & 0 & 0 & 0 \\ -\frac{1}{2} \sin 2 \Theta & \frac{1}{2} \sin 2 \Theta & 0 & -\cos 2 \Theta & 0 & 0 \\ 0 & 0 & 0 & 0 & -\sin \Theta & -\cos \Theta \\ 0 & 0 & 0 & 0 & \cos \Theta & -\sin \Theta\end{array}\right]}_{\mathbf{N}} \Sigma$.

Using Eqs. (6) and (7), we can write,

$\delta \Sigma_{\mathrm{r}}=\mathbf{N M}^{-1} \delta S$

Following the same procedure as in Sect. 2.1.1, we get

$\frac{\left\langle\delta \Sigma_{\mathrm{r}}^{\mathrm{T}} \boldsymbol{\delta} \Sigma_{\mathrm{r}}\right\rangle}{\left\langle\epsilon_{\mathrm{s}}^{2}\right\rangle}=\operatorname{Tr}\left(\mathbf{N M}^{-1}\left(\mathbf{N M}^{-1}\right)^{\mathrm{T}}\right)$.

Equation (14) states that the error variance is dependent on the mean wind direction. In order to make it independent of the mean wind direction, we assume a uniform distribution of the mean wind direction, and estimate the averaged ratio of the error variance. Thus the directionally averaged ratio is,

$$
\begin{aligned}
\frac{\overline{\left\langle\boldsymbol{\delta} \Sigma_{\mathrm{r}}^{\mathrm{T}} \boldsymbol{\delta} \Sigma_{\mathrm{r}}\right\rangle}}{\left\langle\epsilon_{\mathrm{s}}^{2}\right\rangle} & =\frac{1}{2 \pi} \int_{0}^{2 \pi} \operatorname{Tr}\left(\mathbf{N} \mathbf{M}^{-1}\left(\mathbf{N} \mathbf{M}^{-1}\right)^{\mathrm{T}}\right) \mathrm{d} \Theta \\
& =\frac{1}{2 \pi} \int_{0}^{2 \pi} \operatorname{Tr}\left(\mathbf{N} \mathbf{M}^{-1} \mathbf{M}^{-1 \mathrm{~T}} \mathbf{N}^{\mathrm{T}}\right) \mathrm{d} \Theta,
\end{aligned}
$$

where $\ldots{ }^{\Theta}$ denotes directional average. Using the property of matrix trace that it is invariant under cyclic permutations 
we get,

$$
\frac{\overline{\left\langle\delta \Sigma_{\mathrm{r}}^{\mathrm{T}} \boldsymbol{\delta} \Sigma_{\mathrm{r}}\right\rangle}}{\left\langle\epsilon_{\mathrm{s}}^{2}\right\rangle}=\frac{1}{2 \pi} \int_{0}^{2 \pi} \operatorname{Tr}\left(\mathbf{N}^{\mathrm{T}} \mathbf{N} \mathbf{M}^{-1} \mathbf{M}^{-1 \mathrm{~T}}\right) \mathrm{d} \Theta
$$

We can also switch the order between integration and matrix trace, i.e. either we can estimate the trace first and then the integration or vice-versa. Thus,

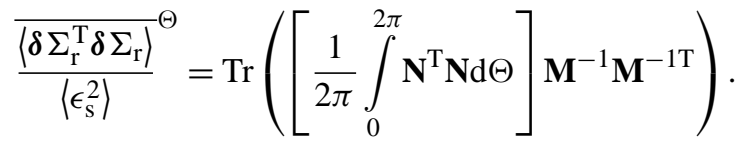

Solving the integral we get,

$$
\frac{\overline{\left\langle\delta \Sigma_{\mathrm{r}}^{\mathrm{T}} \delta \Sigma_{\mathrm{r}}\right\rangle}}{\left\langle\epsilon_{\mathrm{s}}^{2}\right\rangle}=\operatorname{Tr}\left(\left[\begin{array}{cccccc}
\frac{7}{8} & \frac{1}{8} & 0 & 0 & 0 & 0 \\
\frac{1}{8} & \frac{7}{8} & 0 & 0 & 0 & 0 \\
0 & 0 & 1 & 0 & 0 & 0 \\
0 & 0 & 0 & \frac{3}{2} & 0 & 0 \\
0 & 0 & 0 & 0 & 1 & 0 \\
0 & 0 & 0 & 0 & 0 & 1
\end{array}\right] \mathbf{M}^{-1} \mathbf{M}^{-1 \mathrm{~T}}\right) .
$$

The objective is to minimize Eq. (17), subject to the constraints that $\theta$ varies between 0 and $360^{\circ}$ and $\phi$ varies between 0 and $45^{\circ}$. The limit of $45^{\circ}$ for $\phi$ is arbitrary, and is based on the considerations of statistical homogeneity in a horizontal plane. Depending on the type of the terrain the range of $\phi$ could thus be increased or decreased, i.e. if a terrain is horizontally homogeneous over a very large extent, then $\phi$ could be greater than $45^{\circ}$ and vice-versa.

\subsection{Optimizing the objective function}

Equation (17) represents a non-linear optimization problem with 12 unknown variables, i.e. six unknowns are zenith angles $\phi$, and the remaining six are the azimuth angles $\theta$. Owing to the complexity of the optimization problem, an analytical solution of Eq. (17) is not possible. We thus use numerical methods, where either gradient or direct search methods could be used (Rao, 2009). For gradient methods, it is essential that the objective function is differentiable. However we assume that Eq. (17) is a discontinuous function, and hence, we do not use gradient methods. Thus we optimize Eq. (17) using direct search methods only. The main advantage of using direct search methods is that they can be used for discontinuous and non-differentiable functions. The main limitation of such methods is that the found optimum may only be a local optimum.

Different algorithms such as simplex (Nelder and Mead, 1965), simulated annealing (Ingber, 1993) and random search (Rao, 2009) are tested, which result in the optimum angles as given in Table 1, which shows that the optimum configuration consists of five beams equally spaced on the base of a scanning cone and one vertical beam.
Table 1. Optimum six-beam configuration.

\begin{tabular}{lrrrrrr}
\hline Beam no. & 1 & 2 & 3 & 4 & 5 & 6 \\
\hline$\theta^{\circ}$ & 0 & 72 & 144 & 216 & 288 & 288 \\
$\phi^{\circ}$ & 45 & 45 & 45 & 45 & 45 & 0 \\
\hline
\end{tabular}

\section{Description of the measurements}

The six-beam measurements were carried out using the newly developed $1543 \mathrm{~nm}$ pulsed coherent Doppler scanning lidar "long-range WindScanner" (henceforth referred to as WindScanner) at the DTU Wind Energy Department in Denmark. The WindScanner is based on the pulsed lidar Windcube 200 from Leosphere and a dual-axis mirror based steerable scanner head designed by DTU Wind Energy and IPU. The WindScanner is intended for radial velocity measurements from the range of distances between 50 and $6000 \mathrm{~m}$. The current maximum measurement rate is $10 \mathrm{~Hz}$. The maximum number of simultaneous radial velocities acquired at any rate along each line-of-sight is 500. The WindScanner can emit either 400 or 200 ns laser pulses, which are streamed with two corresponding pulse repetition frequencies of 10 and $20 \mathrm{kHz}$ respectively. The energy content of $400 \mathrm{~ns}$ laser pulses is $100 \mu \mathrm{J}$, while the energy content of the $200 \mathrm{~ns}$ laser pulses is half of this value. The scanner head has two rotational degrees of freedom and can rotate around the azimuth and elevation axes, thus it directs the laser pulses into the atmosphere at any combination of azimuth and elevation. The maximum scanner head rotation speed is $50^{\circ} \mathrm{s}^{-1}$, while the maximum acceleration is $100^{\circ} \mathrm{s}^{-2}$. The scanner head can rotate around both axes from 0 to $360^{\circ}$, and the rotation can be endless. The pointing accuracy of the WindScanner is $0.05^{\circ}$. The WindScanner is operated via a remote "master computer" through a UDP/IP and TCP/IP network using the remote sensing communication protocol (RSComPro) (Vasiljevic, 2014).

The measurements were performed at the Danish National Test Center for Large Wind Turbines at Høvsøre, Denmark. Figure 3 shows the location of the test centre in Denmark (see inset in Fig. 3) and the location of the reference $89 \mathrm{~m}$ meteorological (met) mast located at the UTM zone $32 \mathrm{~V}$ $447229 \mathrm{mE}$ and $6256195 \mathrm{~m} \mathrm{~N}$ (WGS84 datum). The high frequency measurements from a reference Ris $\varnothing$ P2564A cup anemometer at $89 \mathrm{~m}$ placed on the top of the met mast are combined with the wind direction measurements from a F2919A Vector W200P wind vane placed in the north direction at $86 \mathrm{~m}$ to deduce $\left\langle u^{\prime 2}\right\rangle$ and $\left\langle v^{\prime 2}\right\rangle$ over a $30 \mathrm{~min}$ period. Since it is not possible to measure the $w$ component using a cup anemometer, comparisons of the corresponding second-order statistics were not possible with the WindScanner measurements. The choice of the 30 min averaging period (instead of the standard 10 min statistics prevalent in the wind energy industry) is made based on the considerations 


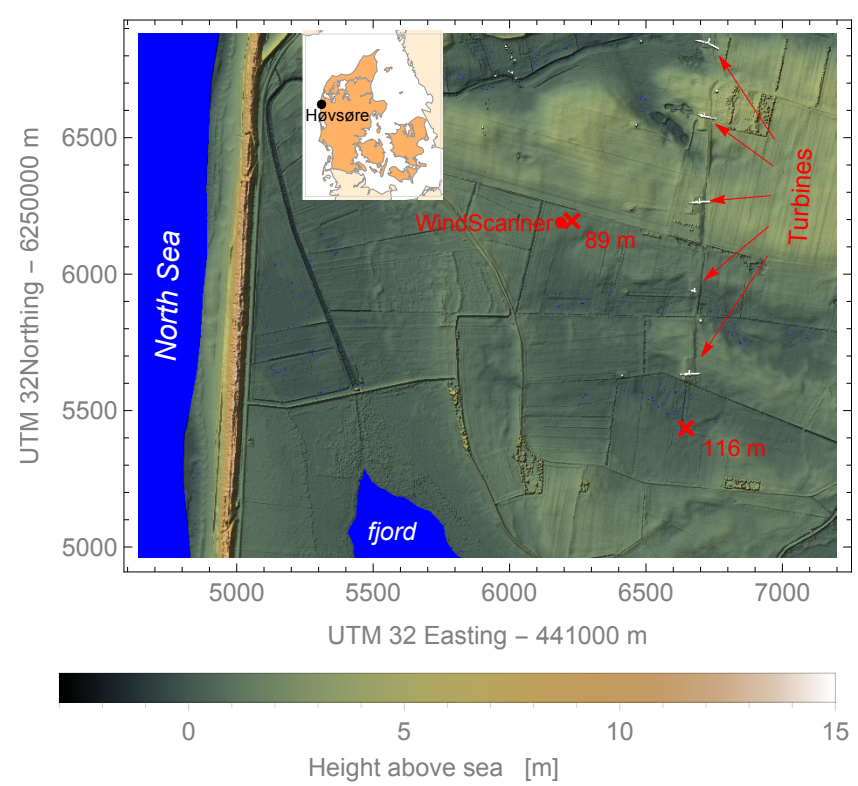

Figure 3. Location of the Høvsøre test centre in Denmark (inset) and details of the site, where the location of the WindScanner and the met masts are shown.

of the random errors in turbulence measurements (Lenschow et al., 1994). The site is about $2 \mathrm{~km}$ from the west coast of Denmark. The eastern sector is characterized by flat homogeneous terrain, and to the south is a lagoon. The WindScanner is placed at the UTM zone $32 \mathrm{~V} 447188 \mathrm{mE}$ and $6256189 \mathrm{~m} \mathrm{~N}$ (WGS84 datum) which is about $41 \mathrm{~m}$ away from the met mast in the west direction. Since the wind turbines are to the east of the WindScanner and the met mast, only the measurements from the western sector $\left(225-315^{\circ}\right)$ are used. Owing to the sudden change in the surface roughness from sea to land in the western sector, we expect the turbulence structure to be influenced by the development of the internal boundary layer, particularly under different atmospheric stabilities. However we do not expect a significant influence on the flow homogeneity in the horizontal direction around the scanning circle, which is one of the key assumptions of the six-beam method.

The duration of the full cycle of the six-beam measurements from the WindScanner was about $15 \mathrm{~s}$. The period of measurement was between 1 and 28 July 2013, where 764 30 min periods were measured. After filtering for data availability within each $30 \mathrm{~min}$ period, where only those periods were chosen with $95 \%$ data, the number of $30 \mathrm{~min}$ periods reduced to 625 . Finally filtering for wind directions to avoid wakes from the wind turbines and the met mast rendered 401 $30 \mathrm{~min}$ periods. The available $30 \mathrm{~min}$ ensembles are further classified into different atmospheric stabilities, characterized by Monin-Obukhov length $L_{\mathrm{MO}}$ based on the intervals given in Table 2 (Sathe et al., 2011a).

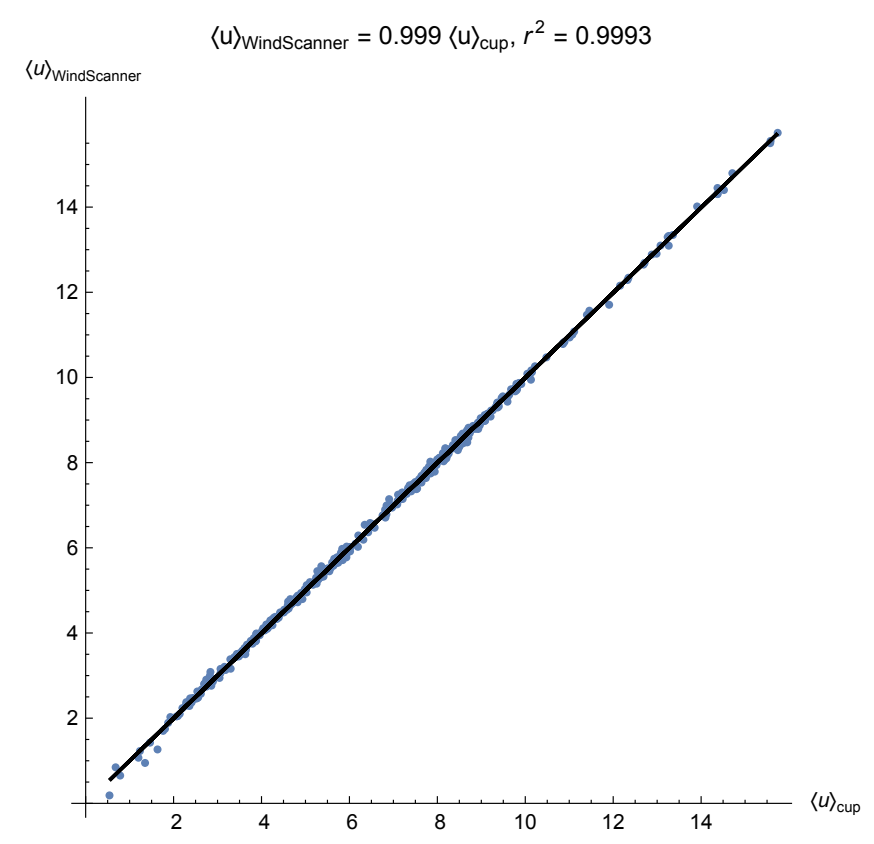

Figure 4. Comparison of the $30 \mathrm{~min}$ mean wind speed between the WindScanner and a cup anemometer using two methods.

Table 2. Classification of atmospheric stability according to Monin-Obukhov length intervals.

\begin{tabular}{ll}
\hline unstable (u) & $-500 \leq L_{\mathrm{MO}} \leq-50 \mathrm{~m}$ \\
neutral (n) & $\left|L_{\mathrm{MO}}\right| \geq 500 \mathrm{~m}$ \\
stable (s) & $10 \leq L_{\mathrm{MO}} \leq 500 \mathrm{~m}$ \\
\hline
\end{tabular}

$L_{\mathrm{MO}}$ is estimated using the eddy covariance method (Kaimal and Finnigan, 1994) from the high frequency $(20 \mathrm{~Hz})$ measurements of a sonic anemometer at $80 \mathrm{~m}$, that is mounted on a $116 \mathrm{~m}$ tall met mast (UTM zone $32 \mathrm{~V}$ $447647 \mathrm{~m} \mathrm{E}$ and $6255435 \mathrm{~m} \mathrm{~N}$ WGS84 datum) in the southeast direction (see Fig. 3). Mathematically, $L_{\mathrm{MO}}$ is given as,

$L_{\mathrm{MO}}=-\frac{u_{*}{ }^{3} \theta_{\mathrm{v}}}{\kappa g \overline{w^{\prime} \theta_{\mathrm{v}}^{\prime}}}$,

where $u_{*}$ is the friction velocity, $\kappa=0.4$ is the von Kármán constant, $g$ is the acceleration due to gravity, $\theta_{\mathrm{v}}$ is the virtual potential temperature, $\ldots$ denotes time average, and $\overline{w^{\prime} \theta_{\mathrm{v}}^{\prime}}$ (covariance of $w$ and $\theta_{\mathrm{v}}$ ) is the virtual kinematic heat flux. $u_{*}$ is estimated as,

$u_{*}=\sqrt[4]{{\overline{u^{\prime} w^{\prime}}}^{2}+{\overline{v^{\prime} w^{\prime}}}^{2}}$,

where $\overline{u^{\prime} w^{\prime}}$ (covariance of $u$ and $w$ ) and $\overline{v^{\prime} w^{\prime}}$ (covariance of $v$ and $w$ ) are the vertical fluxes of the horizontal momentum.

As an initial validation of the accuracy and precision of the WindScanner, the 30 min mean wind speeds were compared with those obtained from the cup anemometer. Figure 4 shows that the WindScanner is very accurate (within $0.1 \%$ ) 

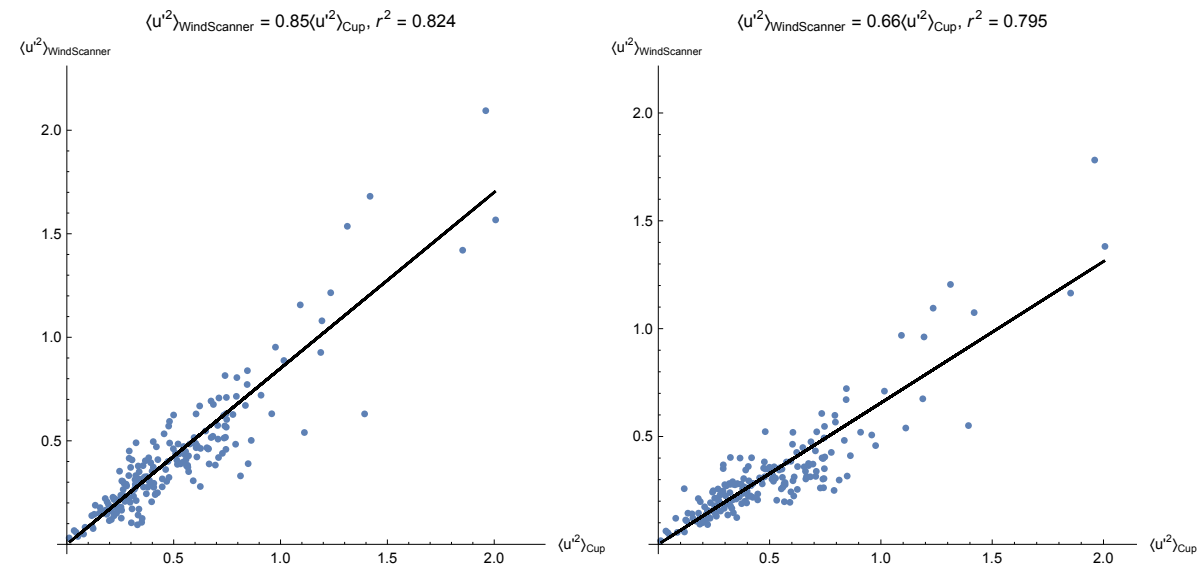

$$
\text { (a) }\left\langle u^{\prime 2}\right\rangle \text { six-beam }
$$

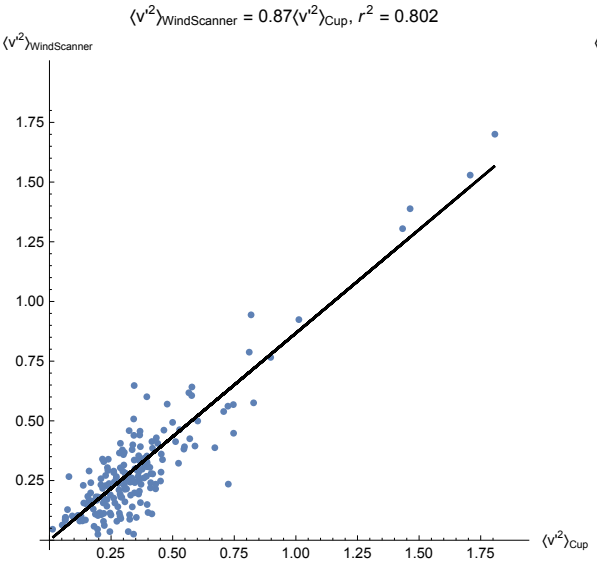

(b) $\left\langle u^{\prime 2}\right\rangle \mathrm{VAD}$

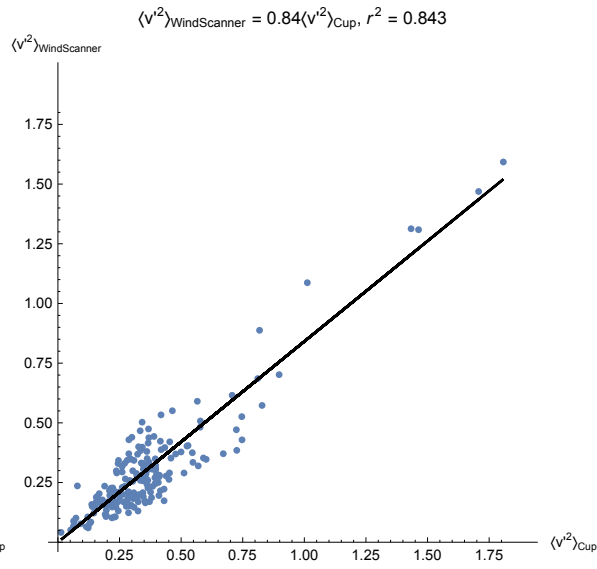

(c) $\left\langle v^{\prime 2}\right\rangle$ six-beam

(d) $\left\langle v^{\prime 2}\right\rangle$ VAD

Figure 5. Comparison of the turbulence statistics under unstable conditions between the WindScanner and the cup anemometer using two methods.

and precise (coefficient of determination, $\left.r^{2} \approx 0.9993\right)$ in measuring the mean wind speeds. For one $30 \mathrm{~min}$ period, the mean radial velocities measured by each of the six beams on the base of the scanning cone were fitted to Eq. (2) in a least squares sense to obtain the 30 min mean wind speed. This procedure was repeated for all $30 \mathrm{~min}$ periods. It is to be noted that the mean wind speed obtained using both methods (six-beam and VAD) is identical, since averaging the radial velocity for each beam, and then making a linear fit to obtain the $u, v$, an $w$ components commute. Such an exercise provided enough confidence to proceed with deducing the turbulence measurements from the WindScanner using both methods.

\section{Turbulence measurements}

Two methods are used to deduce the turbulence statistics from the WindScanner measurements:

1. Six-beam method - for each $30 \mathrm{~min}$ period the measured $S$ vector is used in combination with Eq. (5) to deduce the $\Sigma$ vector. Finally the $\Sigma$ vector is rotated in the mean wind direction for the respective $30 \mathrm{~min}$ period.

2. VAD method - within each $30 \mathrm{~min}$ period the $v_{\mathrm{r}}$ measurements from every single cycle of the six beams are fitted in a least squares sense to Eq. (2) to deduce a 30 min time series of the wind field components. The $\Sigma$ vector is subsequently computed and rotated in the mean wind direction for the respective $30 \mathrm{~min}$ period.

Figure 5 shows the comparison of the turbulence statistics derived from the WindScanner measurements using the six-beam and the VAD methods and those obtained from the cup anemometer under unstable conditions. It is clear that the six-beam method measures more turbulence, about $19 \%$ for $\left\langle u^{\prime 2}\right\rangle$ and $3 \%$ for $\left\langle v^{\prime 2}\right\rangle$ than the VAD method, where the orthogonal least-squares regression is used to fit the cup anemometer measurements. The scatter using both methods is comparable to each other, but there is a slightly more scatter using the six-beam method for $\left\langle v^{\prime 2}\right\rangle$. 

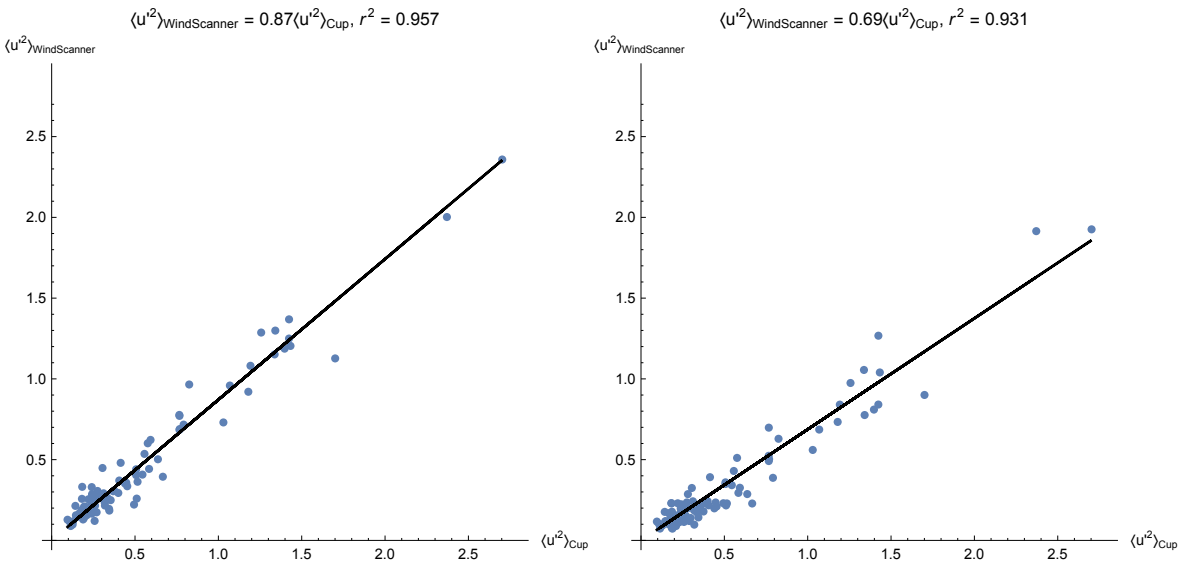

(a) $\left\langle u^{\prime 2}\right\rangle$ six-beam

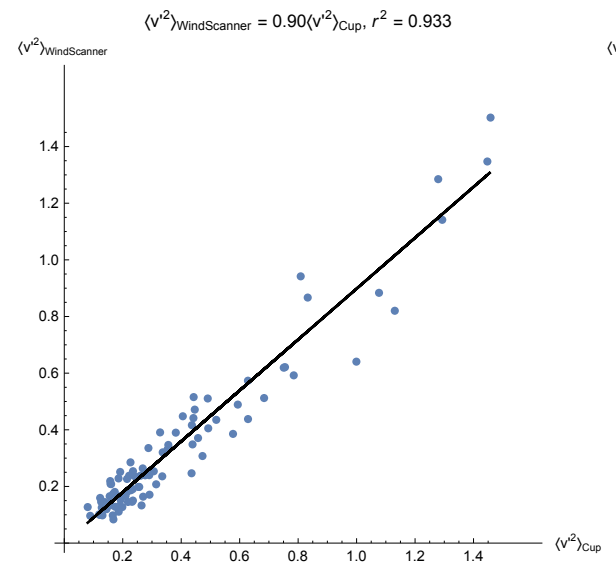

(c) $\left\langle v^{\prime 2}\right\rangle$ six-beam

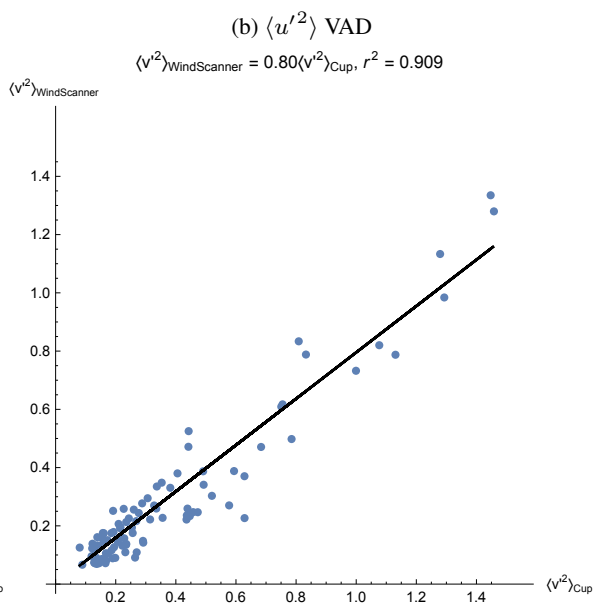

(d) $\left\langle v^{\prime 2}\right\rangle$ VAD

Figure 6. Comparison of the turbulence statistics under neutral conditions between the WindScanner and the cup anemometer using two methods.

Figure 6 shows the same as Fig. 5 but under neutral conditions. As for the unstable conditions, the six-beam method measures more turbulence, about $18 \%$ for $\left\langle u^{\prime 2}\right\rangle$ and $10 \%$ for $\left\langle v^{\prime 2}\right\rangle$ than the VAD method. The scatter using both methods is comparable to each other, with the six-beam method giving a slightly reduced scatter than the VAD method.

Figure 7 shows the same as Fig. 5 but under stable conditions. As for the unstable conditions, the six-beam method measures more turbulence, about $19 \%$ for $\left\langle u^{\prime 2}\right\rangle$ and $4 \%$ for $\left\langle v^{\prime 2}\right\rangle$ than the VAD method. The scatter using both methods is comparable to each other, but there is slightly more scatter using the six-beam method for $\left\langle u^{\prime 2}\right\rangle$.

Thus under all stabilities the six-beam method is closer to the turbulence measurements carried out using the reference cup anemometer. There is however some probe volume averaging using both methods, but this is significantly larger for the VAD method. The probe volume averaging can be observed clearly by comparing the radial velocity spectra, which can be observed in Fig. 6 in Mann et al. (2009), and Fig. 4 in Sjöholm et al. (2009).

\section{Discussion}

From Figs. 5-7 it is clear that using both methods the WindScanner measures more turbulence under stable conditions than under unstable and neutral conditions. This may be contrary to our intuitive understanding, because usually the turbulence scales are much larger under unstable conditions than under stable conditions (Sathe et al., 2013). These results are also contrary to what has been observed by Sathe et al. (2011b) at the same site. However, it is to be noted that Sathe et al. (2011b) used the lidar measurements when the wind was blowing from the eastern direction, whereas in this work we use the measurements when the wind is blowing from the western direction. As described in Sect. 4, in the western sector there is a sudden change of roughness due to the transition from sea to land. As a consequence there is a development of the internal boundary layer (IBL). Also the growth of the IBL depends on atmospheric stability, where under unstable conditions the growth will be faster than under stable conditions. Panofsky and Dutton (1984) state that the growth of the height of the boundary layer is proportional 

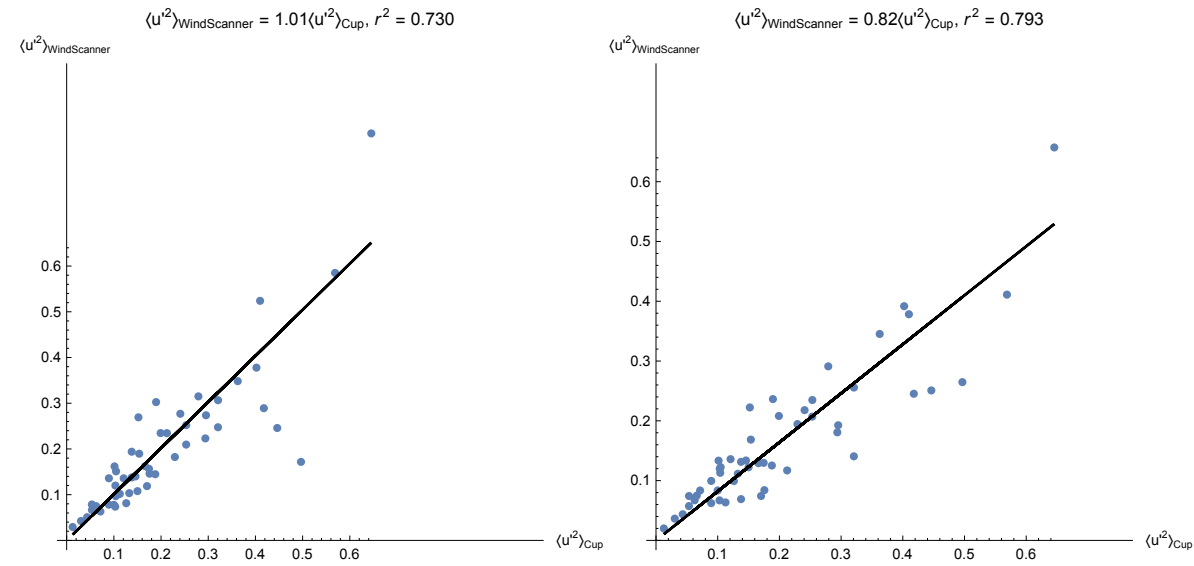

(a) $\left\langle u^{\prime 2}\right\rangle$ six-beam

(b) $\left\langle u^{\prime 2}\right\rangle \mathrm{VAD}$

$\left\langle v^{2}\right\rangle_{\text {Windscanner }}=0.91\left\langle v^{\prime 2}\right\rangle_{\text {cup }}, r^{2}=0.829$

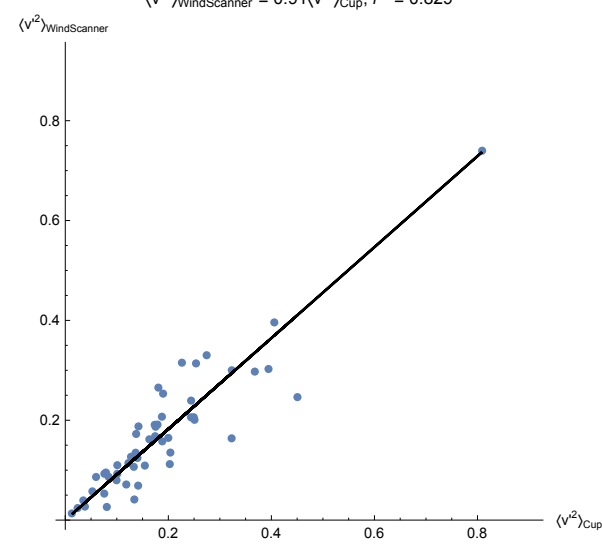

(c) $\left\langle v^{\prime 2}\right\rangle$ six-beam

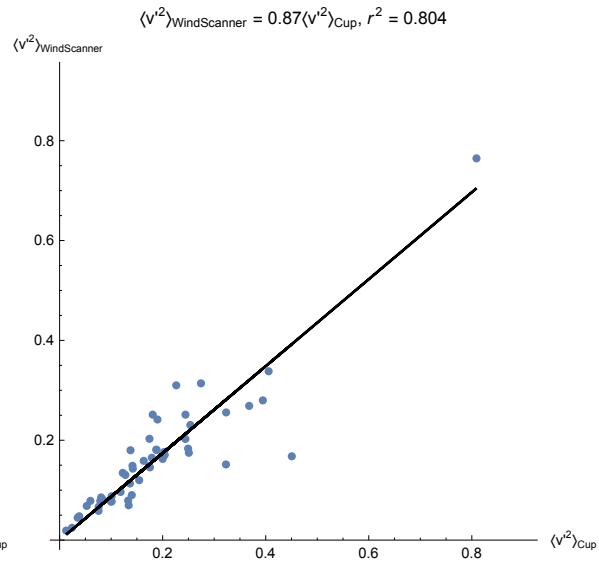

(d) $\left\langle v^{2}\right\rangle$ VAD

Figure 7. Comparison of the turbulence statistics under stable conditions between the WindScanner and the cup anemometer using two methods.

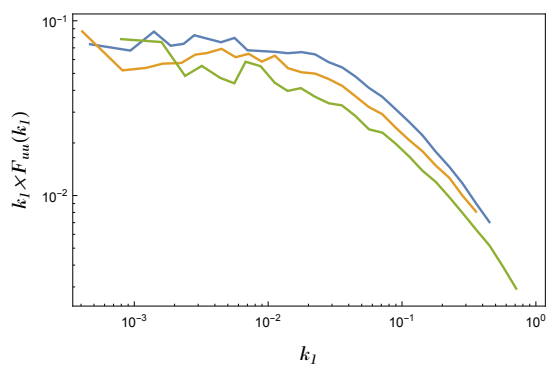

(a) $u$-spectra

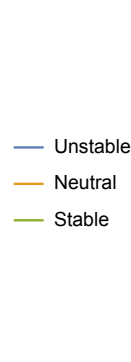

stable

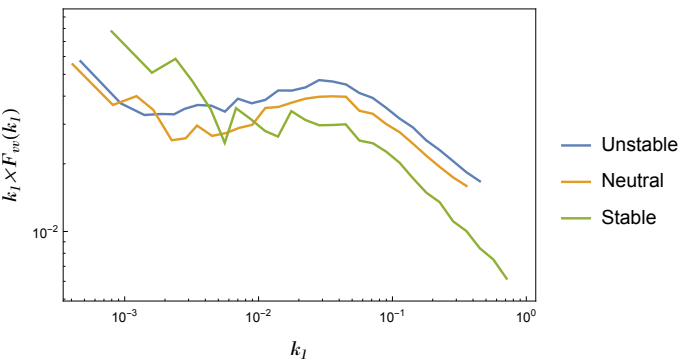

(b) $v$-spectra

Figure 8. Comparison of the $u$ - and $v$-spectra derived from high-frequency cup anemometer measurements under different stability conditions

to the drag coefficient $u_{*} /\langle u\rangle$. And it is well known that the drag coefficient is larger for unstable stratification. Consequently the turbulence scales within the IBL will be smaller as compared to those outside of it. It is then interesting to check whether the WindScanner measures more within the IBL under unstable conditions as compared to the stable conditions.
Figure 8 shows the $u$ and $v$ spectra derived from highfrequency cup anemometer measurements under different stability conditions. If we define the characteristic length scale $\mathcal{L}$ as the length scale corresponding to the maximum spectral energy, it is then clear that the peak of the $v$ spectra is shifted to the right for unstable conditions as compared to the stable conditions. It is not that clear for the $u$ spectra, however the shift of scales to larger wavenumbers un- 
der unstable conditions can still be observed. Thus $\mathcal{L}$ appears smaller under unstable conditions than under stable conditions for the measurements from the western sector used in this work. There is thus more probe volume averaging under unstable conditions than under stable conditions. Hence the WindScanner attenuates the turbulence measurements more under unstable conditions than under stable conditions.

Another interesting observation is that using the VAD method the WindScanner does not measure more turbulence than the reference cup anemometer under any stability condition. This, too, does not agree with that observed by Sathe et al. (2011b), even though the same basic pulsed commercial lidar technology was also used in that work. It is likely due to the fact that in Sathe et al. (2011b) only four beams were used as opposed to six beams, and $\alpha$ was $60^{\circ}$ compared to $45^{\circ}$ used in this work. Therefore the turbulence statistics are not directly comparable with those obtained in Sathe et al. (2011b) even though the same basic commercial lidar was used. Due to the application of the least squares technique on the $v_{\mathrm{r}}$ measurements in this work, there is significant volume averaging around the scanning circle, which is also observed in Sathe et al. (2011b) for a continuous-wave lidar.

\section{Conclusions}

An alternative so-called six-beam method is proposed in place of the standard VAD method to measure atmospheric turbulence using a ground-based wind lidar. The major difference between the two methods is that the six-beam method uses the measurement of the radial velocity variances, whereas the VAD method uses the high frequency measurement of the radial velocity transformed into Cartesian coordinates to deduce turbulence statistics. The scanning configuration of the six-beam method is optimized to minimize the sum of the random errors in the measurement of the components of the $\mathbf{R}$ matrix. In comparison to the reference cup anemometer the six-beam method measures between 85 and $101 \%$ of the reference turbulence, whereas the VAD method measures between 66 and $87 \%$ depending on atmospheric stability. The six-beam method thus overcomes partly the problem of significant probe volume averaging that is otherwise observed by the VAD method.

Furthermore two interesting observations have been made in this study. One is that, using both methods the WindScanner measures more turbulence under stable conditions than under unstable conditions, mainly due to the influence of the internal boundary layer (see Sect. 5). The other is that despite using the same underlying pulsed lidar technology as in Sathe et al. (2011b), the VAD method never measures more turbulence than the reference instrument as was observed in Sathe et al. (2011b) (see Sect. 5 for some explanation). It emphasizes the point that the VAD method is highly sensi- tive to the turbulence structure in the atmosphere, and one must avoid using it to measure atmospheric turbulence.

Future studies must certainly focus on tackling the probe volume averaging effect, which will further strengthen the arguments of using the six-beam method. Smalikho et al. (2005) have provided us with such a framework for pulsed lidars, whereas Mann et al. (2010) have demonstrated it for a continuous-wave lidar.

Acknowledgements. This work is carried out as a part of a research project funded by the Danish Ministry of Science, Innovation and Higher Education - Technology and Production, grant no. 060202486B. The resources provided by the Center for Computational Wind Turbine Aerodynamics and Atmospheric Turbulence funded by the Danish Council for Strategic Research grant no. 09-067216 are also acknowledged. We also thank Erik Juul from DTU Wind Energy department for helping with some sketches.

Edited by: W. Ward

\section{References}

Banakh, V. A. and Smalikho, I. N.: Determination of the turbulent energy dissipation rate from lidar sensing data, Atmos. Ocean. Opt., 10, 295-302, 1997a.

Banakh, V. A. and Smalikho, I. N.: Estimation of the turbulence energy dissipation rate from the pulsed Doppler lidar data, Atmos. Ocean. Opt., 10, 957-965, 1997b.

Banakh, V. A. and Werner, C.: Computer simulation of coherent Doppler lidar measurement of wind velocity and retrieval of turbulent wind statistics, Opt. Eng., 44, 071205, doi:10.1117/1.1955167, 2005.

Banakh, V. A., Smalikho, I. N., Köpp, F., and Werner, C.: Representativeness of wind measurements with a CW Doppler lidar in the atmospheric boundary layer, Appl. Opt., 34, 2055-2067, doi:10.1364/AO.34.002055, 1995a.

Banakh, V. A., Werner, C., Kerkis, N. N., Köpp, F., and Smalikho, I. N.: Turbulence measurements with a CW Doppler lidar in the atmospheric boundary layer, Atmos. Ocean. Opt., 8, 955-959, 1995b.

Banakh, V. A., Werner, C., Köpp, F., and Smalikho, I. N.: Measurement of turbulent energy dissipation rate with a scanning Doppler lidar, Atmos. Ocean. Opt., 9, 849-853, 1996.

Banakh, V. A., Smalikho, I. N., Köpp, F., and Werner, C.: Measurements of turbulent energy dissipation rate with a CW Doppler lidar in the atmospheric boundary layer, J. Atmos. Ocean. Technol., 16, 1044-1061, doi:10.1175/15200426(1999)016<1044:MOTEDR>2.0.CO;2, 1999.

Banakh, V. A., Smalikho, I. N., Pichugina, Y. L., and Brewer, W. A.: Representativeness of measurements of the dissipation rate of turbulence energy by scanning Doppler lidar, Atmos. Ocean. Opt., 23, 48-54, doi:10.1134/S1024856010010100, 2010.

Branlard, E., Pedersen, A. T., Mann, J., Angelou, N., Fischer, A., Mikkelsen, T., Harris, M., Slinger, C., and Montes, B. F.: Retrieving wind statistics from average spectrum of continuouswave lidar, Atmos. Meas. Tech., 6, 1673-1683, doi:10.5194/amt6-1673-2013, 2013. 
Browning, K. A. and Wexler, R.: The determination of kinematic properties of a wind field using a Doppler radar, J. Appl. Meteorol., 7, 105-113, doi:10.1175/15200450(1968)007<0105:TDOKPO>2.0.CO;2, 1968.

Clifton, A., Elliott, D., and Courtney, M.: Ground-Based vertically profiling remote sensing for wind resource assessment, Tech. Rep. 15, Internation Energy Agency, 2013.

Eberhard, W. L., Cupp, R. E., and Healy, K. R.: Doppler lidar measurements of profiles of turbulence and momentum flux, J. Atmos. Ocean. Technol., 6, 809-819, doi:10.1175/15200426(1989)006<0809:DLMOPO>2.0.CO;2, 1989.

Frehlich, R.: Coherent Doppler lidar signal covariance including wind shear and wind turbulence, Appl. Opt., 33, 6472-6481, doi:10.1364/AO.33.006472, 1994.

Frehlich, R.: Effects of wind turbulence on coherent Doppler lidar performance, J. Atmos. Ocean. Technol., 14, 54-75, doi:10.1175/1520-0426(1997)014<0054:EOWTOC>2.0.CO;2, 1997.

Frehlich, R. and Cornman, L.: Estimating spatial velocity statistics with coherent Doppler lidar, J. Atmos. Ocean. Techno., 19, 355366, doi:10.1175/1520-0426-19.3.355, 2002.

Frehlich, R. and Kelley, N.: Measurements of wind and turbulence profiles with scanning Doppler lidar for wind energy applications, IEEE J. Selected Topics Appl. Earth Obs. Remote Sens., 1, 42-47, doi:10.1109/JSTARS.2008.2001758, 2008.

Frehlich, R., Hannon, S. M., and Henderson, S. W.: Performance of a $2-\mu \mathrm{m}$ coherent Doppler lidar for wind measurements, J. Atmos. Ocean. Technol., 11, 1517-1528, doi:10.1175/15200426(1994)011<1517:POACDL>2.0.CO;2, 1994.

Frehlich, R., Hannon, S. M., and Henderson, S. W.: Coherent Doppler lidar measurements of wind field statistics, Bound.-Lay. Meteorol., 86, 233-256, doi:10.1023/A:1000676021745, 1998.

Frehlich, R., Meillier, Y., Jensen, M. L., Balsley, B., and Sharman, R.: Measurements of boundary layer profiles in urban environment, J. Appl. Meteorol. Climatol., 45, 821-837, doi:10.1175/JAM2368.1, 2006.

Frehlich, R., Meillier, Y., and Jensen, M. L.: Measurements of boundary layer profiles with in situ sensors and Doppler lidar, J. Atmos. Ocean. Technol., 25, 1328-1340, doi:10.1175/2007JTECHA963.1, 2008.

Gal-Chen, T., Xu, M., and Eberhard, W. L.: Estimation of atmospheric boundary layer fluxes and other turbulence parameters from Doppler lidar data, J. Geophys. Res., 97, 18409-18423, doi:10.1029/91JD03174, 1992.

Ingber, L.: Simulated Annealing: Practice versus Theory, Math. Comput. Model., 18, 29-57, 1993.

Kaimal, J. C. and Finnigan, J. J.: Atmospheric Boundary Layer Flows - Their Structure and Measurement, chap. Acquisition and processing of atmospheric boundary layer data, 254-281, 7, Oxford University Press, New York, 1994.

Kindler, D., Oldroyd, A., Macaskill, A., and Finch, D.: An eight month test campaign of the QinetiQ ZephIR system: Preliminary results, Meteorol. Z., 16, 479-489, doi:10.1127/09412948/2007/0226, 2007.

Kropfli, R. A.: Single Doppler radar measurement of turbulence profiles in the convective boundary layer, J. Atmos. Ocean. Technol., 3, 305-314, doi:10.1175/15200426(1986)003<0305:SDRMOT>2.0.CO;2, 1986.
Lenschow, D. H., Mann, J., and Kristensen, L.: How long is long enough when measuring fluxes and other turbulence statistics?, J. Atmos. Ocean. Technol., 11, 661-673, doi:10.1175/15200426(1994)011<0661:HLILEW>2.0.CO;2, 1994.

Lhermitte, R. M.: Note on wind variability with Doppler radar, J. Atmos. Sci., 19, 343-346, doi:10.1175/15200469(1962)019<0343:NOWVWD>2.0.CO;2, 1962.

Lhermitte, R. M.: Note on the observation of small-scale atmospheric turbulence by Doppler radar techniques, Radio Sci., 4, 1241-1246, doi:10.1029/RS004i012p01241, 1969.

Mann, J., Cariou, J., Courtney, M., Parmentier, R., Mikkelsen, T., Wagner, R., Lindelow, P., Sjöholm, M., and Enevoldsen, K.: Comparison of $3 \mathrm{D}$ turbulence measurements using three staring wind lidars and a sonic anemometer, Meteorol. Z., 18, 135-140, doi:10.1127/0941-2948/2009/0370, 2009.

Mann, J., Peña, A., Bingöl, F., Wagner, R., and Courtney, M. S.: Lidar scanning of momentum flux in and above the surface layer, J. Atmos. Ocean. Technol., 27, 792-806, doi:10.1175/2010JTECHA1389.1, 2010.

Nelder, J. A. and Mead, R.: A Simplex Method for Function Minimization, Computer J., 7, 308-313, 1965.

Panofsky, H. A. and Dutton, J. A.: Atmospheric Turbulence, John Wiley \& Sons, New York, 1984.

Peña, A., Hasager, C. B., Gryning, S.-E., Courtney, M., Antoniou, I., and Mikkelsen, T.: Offshore Wind Profiling Using Light Detection and Ranging Measurements, Wind Energy, 12, 105-124, doi:10.1002/we.283, 2009.

Rao, S.: Engineering Optimization: Theory and Practice, John Wiley and Sons Inc., Hoboken, New Jersey, 4th Edn., 2009.

Sathe, A. and Mann, J.: Measurement of turbulence spectra using scanning pulsed wind lidars, J. Geophys. Res., 117, D01201, doi:10.1029/2011JD016786, 2012.

Sathe, A. and Mann, J.: A review of turbulence measurements using ground-based wind lidars, Atmos. Meas. Tech., 6, 3147-3167, doi:10.5194/amt-6-3147-2013, 2013.

Sathe, A., Gryning, S.-E., and Peña, A.: Comparison of the atmospheric stability and wind profiles at two wind farm sites over a long marine fetch in the North Sea, Wind Energy, 14, 767-780, doi:10.1002/we.456, 2011a.

Sathe, A., Mann, J., Gottschall, J., and Courtney, M. S.: Can wind lidars measure turbulence?, J. Atmos. Ocean. Technol., 28, 853868, doi:10.1175/JTECH-D-10-05004.1, $2011 \mathrm{~b}$.

Sathe, A., Mann, J., Barlas, T., Bierbooms, W. A. A. M., and van Bussel, G. J. W.: Influence of atmospheric stability on wind turbine loads, Wind Energy, 16, 1013-1032, doi:10.1002/we.1528, 2013.

Sjöholm, M., Mikkelsen, T., Mann, J., Enevoldsen, K., and Courtney, M.: Spatial averaging-effects on turbulence measured by a continuous-wave coherent lidar, Meteorol. Z., 18, 281-287, doi:10.1127/0941-2948/2009/0379, 2009.

Smalikho, I., Kopp, F., and Rahm, S.: Measurement of atmospheric turbulence by $2-\mu \mathrm{m}$ Doppler lidar, J. Atmos. Ocean. Technol., 22, 1733-1747, doi:10.1175/JTECH1815.1, 2005.

Smalikho, I. N.: On measurement of dissipation rate of the turbulent energy with a CW Doppler lidar, Atmos. Ocean. Opt., 8, 788793, 1995

Smith, D. A., Harris, M., Coffey, A. S., Mikkelsen, T., Jørgensen, H. E., Mann, J., and Danielian, R.: Wind Lidar Evaluation at 
the Danish Wind Test Site in Høvsøre, Wind Energy, 9, 87-93, doi:10.1002/we.193, 2006.

Vasiljevic, N.: A time-space synchronization of coherent Doppler scanning lidars for 3D measurements of wind fields, Ph.D. Thesis PhD-0027 (EN), Technical University of Denmark, 2014.
Wagner, R., Courtney, M., Gottschall, J., and Lindelöw-Marsden, P.: Accounting for the speed shear in wind turbine power performance measurement, Wind Energy, 14, 993-1004, doi:10.1002/we.509, 2011.

Wilson, D. A.: Doppler Radar Studies of Boundary Layer Wind Profiles and Turbulence in Snow Conditions, in: Proc. 14th Conference on Radar Meteorology, 191-196, Tucson, USA, 1970. 